\title{
Architecture of LTE-WIFI Integration for SDN Towards IP Systems
}

\author{
Chen Yange, Zhang Zhili, Du Genyuan, Chen Shangbo \\ College of Information Technology, Xuchang University \\ chenyange_ok@126.com
}

\begin{abstract}
Against the background of a surge in software-defined network (SDN) as well as urgent demands for LTE-WIFI internetworking and IPv6 network integration, the paper integrates LTE mobile telecommunication technology, wireless local area network and $5 G$ (the fifth generation mobile communication system) into one, in an attempt to complete routing, distribution, and transmission of different radio frequency signals (i.e. LTE, WIFI) on the SDN basis. As a result, large-area coverage of LTE-WIFI network signals is realized in a way that meeting the demands of reliability, safety, flexibility and extendibility for mobile internet. What is more, different complementary wireless telecommunication systems are closed combined with each other. Through the use of SDN integration technology, the paper designs network integration architecture of IP-LTE mobile internet and WIFI, and also studies on the application of SDN-based IP optical integration network, which has broad prospects.
\end{abstract}

Keywords: Software Defined Network; Long Term Evolution; Wireless Local Area Networks; network convergence

\section{Introduction}

The wide-area coverage is allowed full play in the LTE network technology, while WIFI network technology achieves localized high-speed data transmission. Considering their complementary edges, how to technically realize effective integration of LTE with WIFI is a pressing problem. The global interworking of Internet of Things business and the mobile internet business, which is still at a preliminary stage, hence, are endowed with broad prospects and rare future opportunities in relation to its business application [1].

As the principal mode of Internet of Things, LTE-WIFI integration involves diversified business types. However, the corresponding models adopted at the current stage are easy to underuse network resources and information due to their small memory and high frequency. As a result, the development of Internet of Things is impeded. Against this backdrop, the integration of LTE with Internet of Things performs better than any of the separate ones, and can better realize high-efficiency, rational data processing [2].

In this connection, any breakthrough made in key technologies of interworking of mobile internet and WIFI will help to extend application scopes into new industry space, with provision of better service. It can also stimulate information consumption, which is the reliable foundation of construction of "connected life".

The paper takes advantage of both cellular network and local area network (LAN) to build up a SDN-based LTE-WIFI integration network, in an attempt to address the problem of internet reuse or network flow waste during switch between mobile network and WIFI. With this integration network, the actual distance from input ends to output ends is phenomenally shortened, the follow-up improvement of network coverage and service quality leads to better performance on end users. What is more, system capacity augments brings significant increment in power efficiency and frequency spectrum efficiency. Meanwhile, there are no more difficulties in the automatic switch between 
mobile network and WIFI network.

\section{Dynamic Analysis of Research Status and Research Progress}

The development of 4G-LTE-based 5G (the fifth generation mobile communication system) is undoubtedly centered on user experience, for which such fields as terminal, wireless network, business and network will undergo integration and innovation. At the same time, a revolution in users' capability of information perception, acquirement, participation and control arises from the use of 5G. In the future, 5G will reflect the advantages of cellular network and LAN, and bring us a more intelligent and friendly environment with the establishment of mobile network and WIFI integration [3]. Therefore, studies on relevant key technologies have the practical significance to the entire development of information industry [4].

$5 \mathrm{G}$ is differentiated from the fourth generation communication system or LTE (Long term evolution), in general because the technology is an integration of existing wireless telecommunication technologies rather than a single wireless technology. The use of 5G will definitely open into the coexistence of various network types and communication modes. 5G wireless transmission acts as the core technology of mobile cloud computing, through which the computation and data to be processed are transported from a mobile device to the cloud. In this way, not only can the shackles of insufficient native resources been thrown off, but also the level of device consumption is reduced. $5 \mathrm{G}$ will satisfy the requirements of super high network flow density, super high connection density, and super high mobility. In addition, the use of $5 \mathrm{G}$ provides users with the opportunity to experience some premium services such as high-definition videos, virtual reality, augmented reality, cloud desktop and online games. With 5G, multiple heterogeneous networks can coexist with each other, and various types of wireless access technologies are used by mobile devices to $\log$ on the cloud. With $5 \mathrm{G}$, network construction and network operation can be much more energy-saving and cost-effective [1].

Either LTE or WIFI in relation to 5G can independently improve the efficiency and performance of wireless communication. However, when they are integrated, strong interference appears [5]. Currently, the heterogeneous LTE-WLAN multi-radio technology has been applied to commercial intelligent cellphones in the form of broadcast. Multi-radio-enabled intelligent devices are equipped with LTE wireless interfaces and WIFI interfaces, which realizes seamless mobility and bandwidth aggregation [17]. Some surveys are conducted from the perspective of overall network resource distribution as well as based on heterogeneous LTE-WLAN bandwidth, and the result shows that it has become an important network utilization mode to enhance heterogeneity in equipment bandwidth through a centralized allocation of multi-radio users [6].

Jin X et al. propose a scalable and flexible cellular core network architecture that LTE cellular network based on Software Defined Network (SDN) will be the development trend of the next generation core network architecture [18]. Ali-Ahmad $\mathrm{H}$ et al. propose a dense network of LTE and WLAN based on SDN [19]. Saehoon Kang and Wonyong Yoon propose SDN-based resource allocation for heterogeneous LTE and WLAN multi-radio networks [7]. Nagaraj et al. propose smart traffic handling for scalable software EPC that offloading flows from the EPC to other IP transport networks based on flow classification [20]. Heinonen et al. propose Dynamic tunnel switching for SDN-Based Cellular core networks [8].

Mendonca et al. examine the probability of software defined networking in heterogeneous networked environments which include heterogeneous wired networks, wireless networks based on infrastructure, and wireless networks without infrastructure [21]. Ali-Ahmad H, Cicconetti C et al. propose SDN-based network architecture for extremely dense wireless networks $[19,22]$. Rasha Alkhansa et al. propose LTE-WiFi Carrier Aggregation for Future 5G Systems: A Feasibility Study and Research Challenges 
[23]. Through the study of relevant results, it is better to carry out the research on the integration of $5 \mathrm{G}$ ultra dense networks and internet of things. On this basis, this paper studies the design of LTE-WIFI network deployment based on SDN for the IP evolution.

\section{The SDN-based Integration Scheme Towards IP Architecture}

Among the three technological solutions that are defined by 5G core network, which has network function virtualization (NFV), SDN and cloud RAN (C-RAN)[9].We adopt the SDN-NFV integration one due to its provision of dynamic, prompt interaction chances and its ability to vary network service mode. The SDN decouples the network control and forwarding functions enabling the network control to become directly programmable and the underlying infrastructure to be abstracted for applications and network services. NFV is a network architecture concept that uses the technologies of IT virtualization to virtualize entire classes of network node functions into building blocks that may connect, or chain together, to create communication services. The integration of SDN and NFV helps realize manageable, dynamic, and adaptive network [10].

SDN and NFV combination of the program, SDN controller go on the unified management and centralized control for forwarding equipment, OpenFlow controller is responsible for generating, maintenance and issued of network flow table; OpenFlow switch is responsible for forwarding data, has data flow table, safe passage and OpenFlow three components [11].SDN controller can realize the conversion between IPv4 and IPv6 protocol, and translation function has software realization, software based network make quickly the deployment of DS-lite, NAT64 and other IPv4/v6 transition technologies; Mutual access for IPv4 network and IPv6 network, according to the requirement of forwarding to different protocol conversion gateway to deal. NFV application in data center edge, if some functions cannot be realized in data center, can use other data center resources, data center resources can provide high-speed user requirements and processing capabilities.

\subsection{Dual-stack Core SDN Integration Mode}

Currently we need to study the development of dual-stack core SDN fusion network which should be designed into IPv4/IPv6 core SDN integration mode. The stub network evolving into access point (AP) nodes and LTE nodes are transmitted to multiprotocol gateway or wireless bridge in the LTE base station. Meanwhile, considering that the LTE evolved node B (eNB) in LTE-WIFI integration is the simple P-GW, AP falls within the area of LTE eNB [7], the wireless network with an integration of intelligent wireless switch with intelligent LTE-WIFI is formed, towards which data are transmitted. Sensors and wireless devices of the stub network have partial IP addresses. The access sub-network as IPv4 network or IPv6 network is transmitted to IPv4/IPv6 core SDN according to the initial protocol. It then enters the software platform in the SDN controller, and is distributed to different protocol conversion module for network processing. The corresponding fusion mode is shown in Figure 1. 


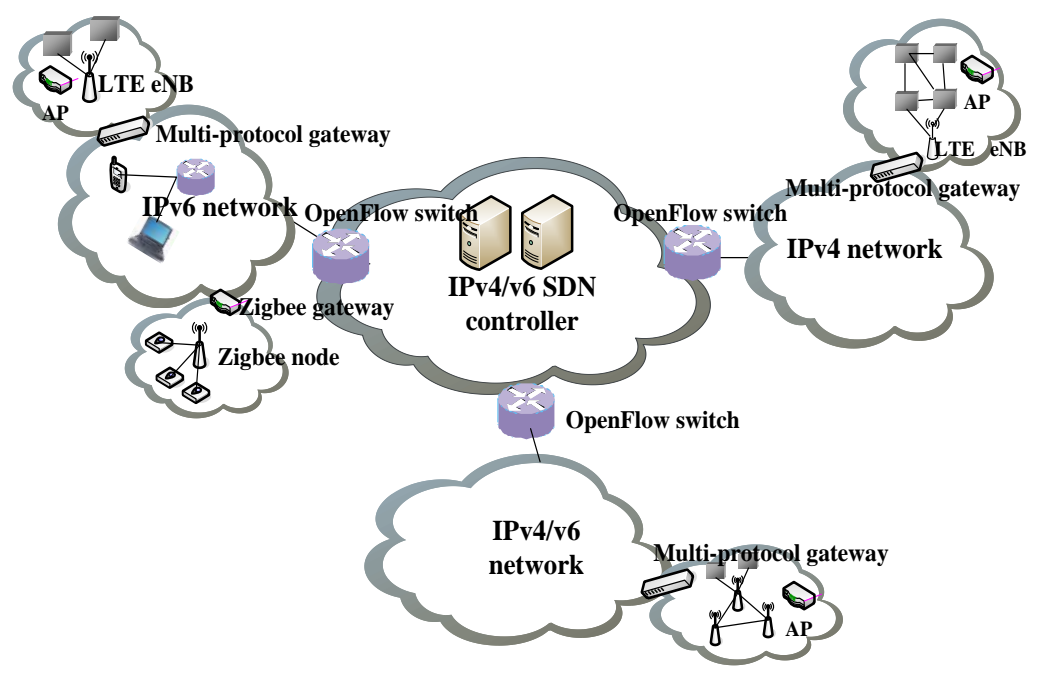

Figure 1. LTE-WIFI Fusion Mode of Dual-stack Core SDN Network

When the host of IPv4 network communicates with IPv6 network, the IPv4 host sends data packets to SDN controller via OpenFlow switch. Specifically, IPv4 address is carried in the IPv6 header in the charge of SDN controller which transports the data to IPv6 network via the gateway or the OpenFlow switch. After the communication, data in IPv6 host retraces its way to IPv4 network, which marks the completion of IPv4-to-IPv6 communication. For IPv6-to-IPv4 communication, IPv6 data packets are sent to the SDN controller via the gateway or the OpenFlow switch. Then, protocol conversion modules such as NAT64 are used to translate the data packets so as to map IPv6 address to IPv4 address. The mapped data packets are transmitted to IPv4 network via OpenFlow switch, and mapped back to IPv6 address during its retracing. If the access sub-network is IPv4/IPv6 dual-stack network, any protocol in the SDN controller can be used to realize its network communication.

\subsection{IPv6 Core SDN Integration Mode}

As the internet evolves in a nonstop manner, IPv6 core SDN controller network is to become the core network, and the network section will adopt IP optical integration network that is based on SDN. Sensors or wireless devices in the stub network collect data via LTE base station, WIFI or Zigbee nodes. For LTE base station, LTE-WIFI data returns to the intelligent wireless-controlled multi-protocol gateway, and the aggregated data is sent to the access network. Automatic distribution of IPv6 address is required for WIFI to realize networking of access devices. The radio over fiber (ROF) technology can be used to construct a hybrid optical radio frequency system, on which different access networks are required for integration, such as LTE (4G) mobile telecommunication network, WLAN, and MESH, as shown in Figure 2. 


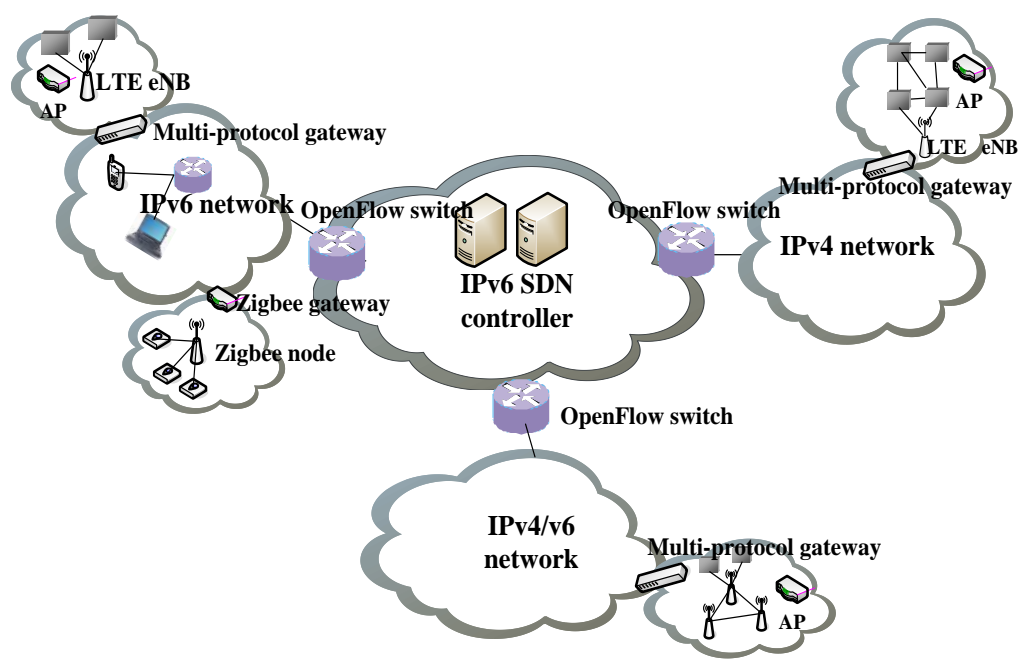

Figure 2. LTE-WIFI Fusion Mode of IPv6 Core SDN Network

\subsection{Full IPv6 SDN Integration Mode}

Internet of Things itself and its access subnetwork will finally turns into full IPv6 SDN network, where all the access materials and devices undergo network transmission in the form of IPv6 and are automatically distributed to lightweight IPv6 address. The SDN-controller-based full IPv6 network serves as the core network, through which all sensors and wireless devices in the stub network and access network are endowed with IP and can automatically organize networks. What is more, LTE and WIFI have been completely integrated with each other, as shown in Figure 3. Mobile terminal devices select optimal WIFI integration schemes as required. At present, the proposed LTE-WIFI fusion scheme has Dynamic Backhaul Sensitive Network Selection Schemes [24], Access Network Discovery and Selection Function (ANDSF) [12] etc. They operate with the LTE technology in areas beyond the coverage of the WIFI network, but automatically connect to WIFI if possible. In this way, these devices realize optimal user throughput, and save data flow $[25,26]$.

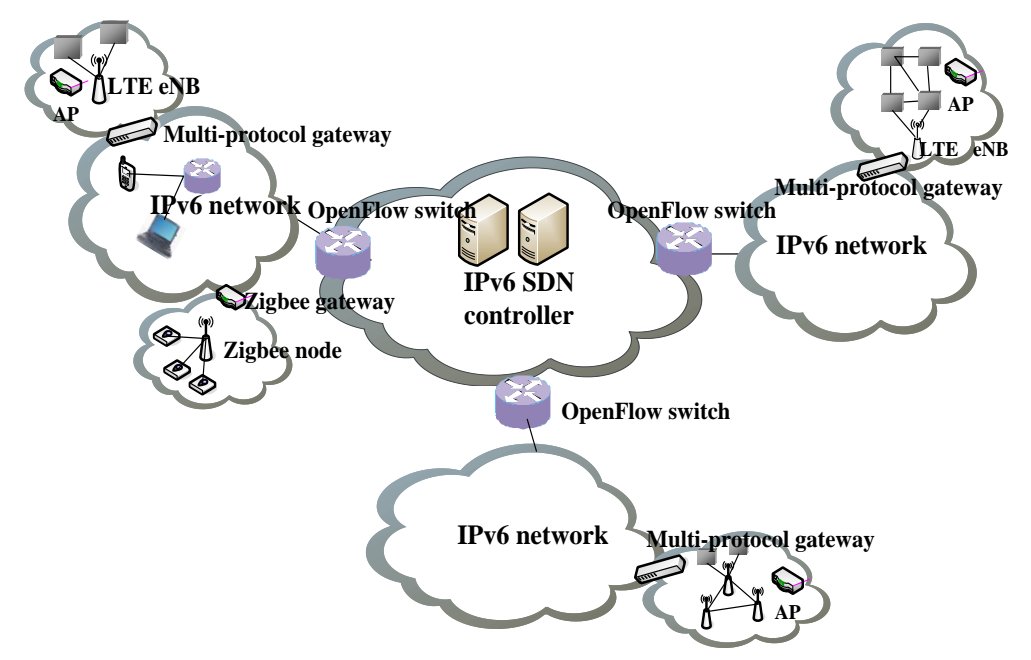

Figure 3. LTE-WIFI Fusion Mode of full IPv6 SDN Network

Through the said analysis, the paper obtains the overall architecture of IPv6-based SDN, as shown in Figure 4. Lightweight of IPv6 is conducted on IP-based heterogeneous network. Related protocols such as Internet of Things protocol stack and the internet 
protocol stack are deployed on the gateway of Internet of Things, as a way to deploy multi-protocol gateways and to match lightweight IPv6 with normal IPv6. SDN-based IPv6 remains to be adopted by the core layer and the distribution layer, to the advantage of decoupling the network controlling and forwarding functions.

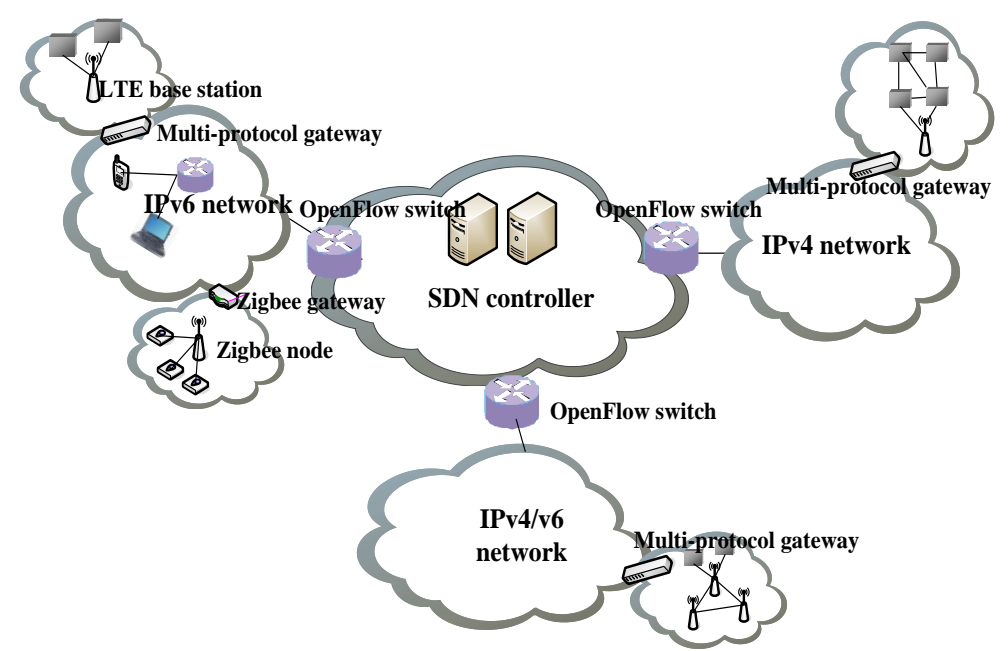

Figure 4. LTE-WIFI Fusion Model of SDN Controller

As can be seen from Figure 4, SDN controller core network is the core network of Internet of Things, which is connected to the access network via OpenFlow switch. The core convergence layer employs SDN-based IPv4/IPv6 network to operate such IPv6 transition technologies as protocol translation and DS-Lite in the form of software. Besides, address resources are managed as a whole. OpenFlow switch of network devices uses OpenFlow protocol, to which new flow tables can be added, for instance, IPv6 message headers can be added to IPv4 data packages and vice versa [13]. In turn, such additional message headers can be gotten rid of in the process of decapsulation, making it possible to identify the protocol traversing networks. OpenFlow switch accounts for data forwarding, which can separate not only control plane from data plane but Protocol Translation from data forwarding [27].

When a cellphone moves within or beyond the scope of WIFI, it voluntarily selects from mobile network and WIFI. WIFI is the priority because the nearby base station of mobile network closes automatically within the scope of WIFI and opens for connection when WIFI signal fails to be detected. Therefore, the effort of manual switch between WIFI and mobile network is saved, so that improving network utilization efficiency and reducing network flow dissipation.

\section{SDN-based IP Optical Integration Network Deployment}

For the said network architecture of IPv6 network evolution, according to the conditions of present networks and its future progress, the paper deploys SDN-based IP optical integration network, and conducts in-depth research on the deployment from the aspects of both the core aggregation network and the access network in relation to Internet of Things.

\subsection{The Deployment of Core Layer and Aggregation Layer}

IP optical aggregation multi-controller is used in the core layer. The IP layer and the optical layer have their own controllers to operate [14]. OpenFlow protocol is used in the heterogeneous network consisting of IPv4/IPv6 network and optical network. The access nodes in the controllers write in corresponding network configuration data and topological 
data. The IP optical aggregation controller aggregates data from IPv4/IPv6 network and optical network for overall control. The IP layer controller is in charge of IPv4/IPv6 network, IPv4 network and IPv6 network, while the optical controller manages the optical network. The IP layer controller and the optical controller are both controlled by the IP optical aggregation controller, which constitutes SDN-based IP optical integration network, as shown in Figure 5. OpenFlow protocol is used to acquire immediate network conditions, based on which the next forwarding method of the IP data flow is determined in a flexible way [15].

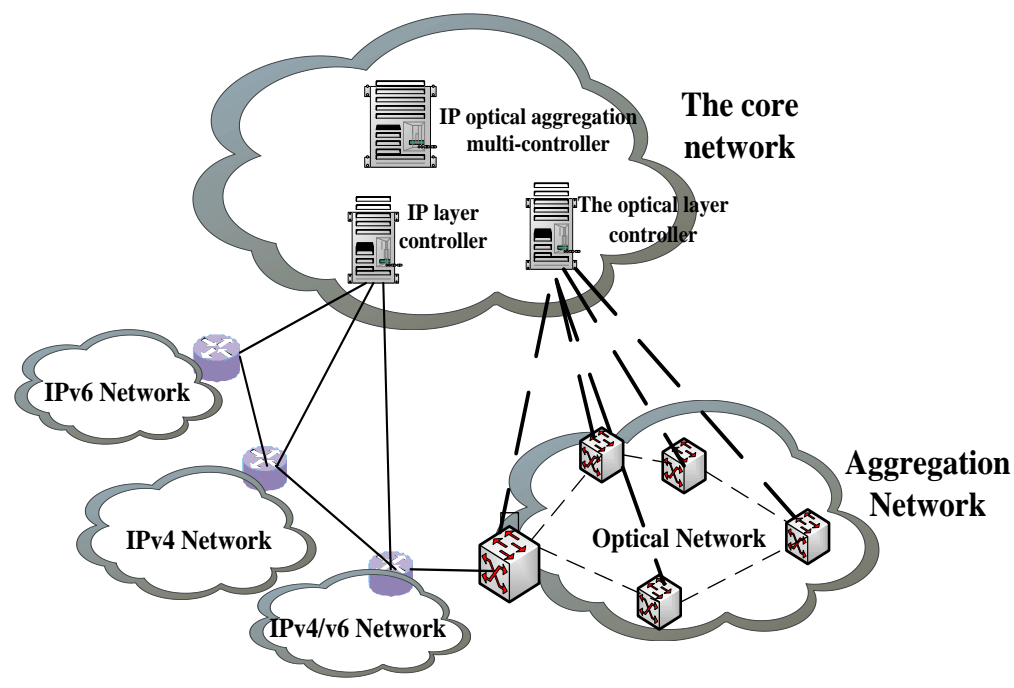

Figure 5. SDN-based IP Optical Integration Network

SDN-based IP optical integration network also decouples the network controlling and forwarding functions, where IP layer control and optical layer control are divided first and then aggregated. In this way, the decoupling between network controlling functions and data forwarding functions are realized, and the localized and overall communication controls, mobile management and service quality guarantee are achieved. What is more, the paper studies on construction of the open interface towards business network capacity [16], including control logic, arrangement as required, and capacity opening function in Control plane. The research into forwarding functions is based on universal hardware platform (gateway, content distribution, service acceleration, etc.) in forwarding plane, and operates in a uniform, flexible, and efficient manner. 


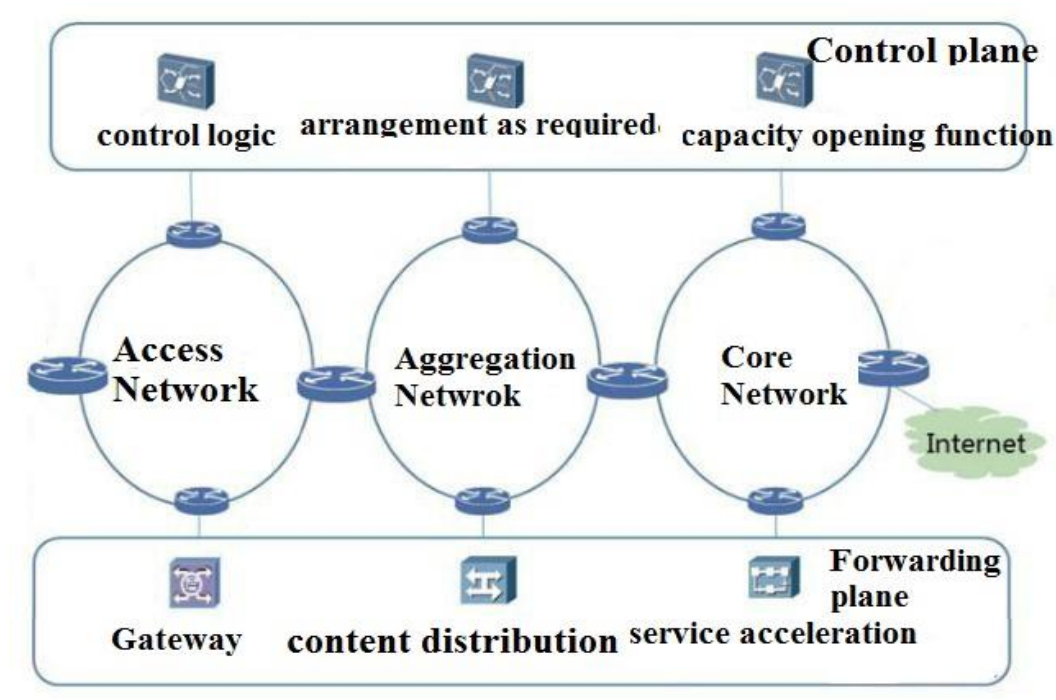

Figure 6. Function Analysis of IP Optical Fusion Network

\subsection{The Deployment of Access Network}

The access approach of WIFI, C-RAN, D2D, MESH and other access networks are mainly studied in this section. According to the result, there are two network architectures for wireless access which adapt to all kinds of return channels: centralized access and distributive access, as shown in Figure 7.

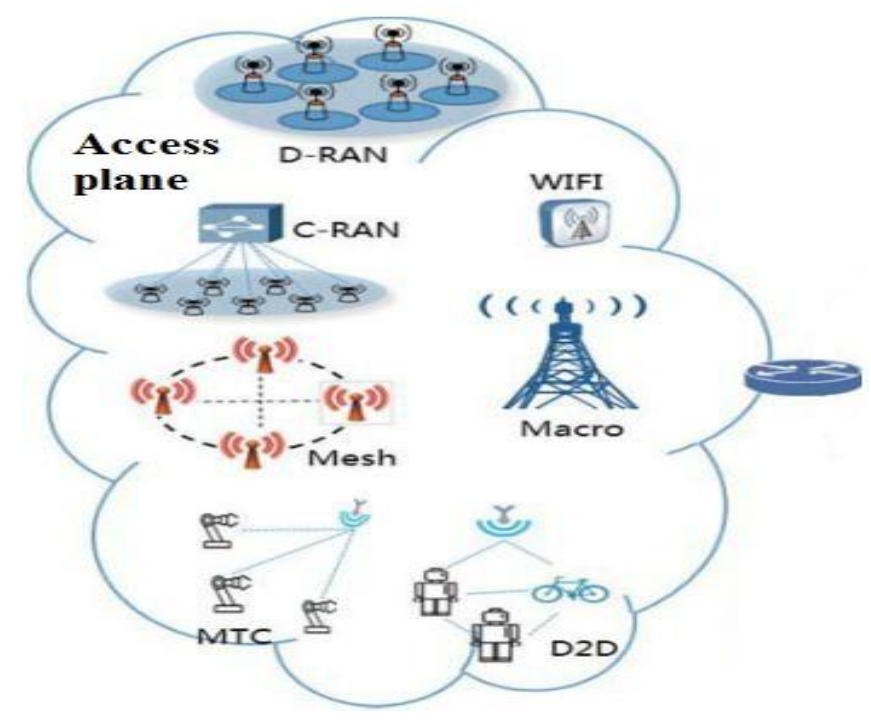

Figure 7. Access Network of SDN-based IP Optical Integration Network

\section{Conclusion}

Network integration is one of the key technologies to research in Internet of Things. The present-day focus of related heterogeneous network integration rests in IP network and SDN. How to realize the effective integration of LTE with WIFI is a pressing problem. Considering that current studies on LTE-WIFI integration is limited to SDN or mobile network integration, the paper conducts in-depth research into architecture of LTE-WIFI integration SDN towards IP systems. The network architecture that dual-stack SDN core network evolves into full IPv6 SDN core network is analyzed in the paper, based on which the network integration model that deals with different stages of internet core 
network and stub network evolution is proposed in a systematic way. Meanwhile, the SDN-based IP optimal integration network application is studied herein, which lays the foundation for follow-up research into interconnection and interworking of Internet of Things.

\section{Acknowledgements}

This work is supported by three projects are highly appreciated. The projects have the next generation network technology innovation project of ministry of education (NGII20150610), Science and Technology project of Education Department of Henan Province (15A510012、17A520055).

\section{References}

[1] D. Ming and Q. Yuan, "Research of the integration and development of Internet of things and mobile Internet", Telecommunication network technology, vol. 8, (2013).

[2] H. L. Yang, "Talking about the combination of LTE wireless communication technology and Internet of things technology”, Heilongjiang Science and Technology Information, vol. 5, (2016).

[3] W. B. Wang, "The design and implementation of digital community network education platform based on mobile Internet", Master Thesis of Electronic Science and Technology University, vol. 9, (2014).

[4] J. Z. Luo, W. J. Wu and M. Yang, "Mobile Internet: terminal, network and service", Journal of Computer Science, vol. 34, no. 11, (2011).

[5] X. M. Liu, "Analysis on the coexistence interference between WiFi and LTE in mobile terminal", New communication in China, vol. 3, (2015).

[6] R. Mahindra, H. Viswanathan, K. Sundaresan, M. Y. Arslan and S. Rangarajan, "A practical traffic management system for integrated LTE-WiFi Networks", In: ACM MobiCom, (2014), pp. 189-200.

[7] S. Kang and W. Yoon, "SDN-based resource allocation for heterogeneous LTE and WLAN multi-radio networks", Journal Supercomput, Springer Science, vol. 72, no. 4, (2016), pp. 1342-1362.

[8] J. Heinonen, T. Partti, M. Kallio, K. Lappalainen, H. Flinck adn J. Hillo, "Dynamic tunnel switching for SDN-Based Cellular core networks. In: Workshop on all things cellular, (2014), pp. 27-32.

[9] L. Gavrilovska, V. Rakovic and V. Atanasovski, "Vision Towards 5G: Technical Requirements and Potential Enablers", Wireless Pers Commun, vol. 87, no. 3, (2016), pp. 731-757.

[10] N. N. He and Y. H. Xue, "Elastic network architecture and its application based on sdn/nfv technology", Telecommunication network technology, vol. 4, (2015).

[11] M. T. Yang, "SDN network simulation design of openflow and DoS attack detection", Master's degree thesis of Beijing University of Posts and Telecommunications, vol. 1, (2015).

[12] D. H. Hagos, "The performance of network-controlled mobile data offloading from LTE to WIFI networks", Telecommun Syst, vol. 61, no. 4, (2016), pp. 675-694.

[13] S. J. Ma and D. Y. Hu, "Implementation of dynamic traffic control for multi service based on IP fusion switching technology based on SDN", Science in China: Information Science, vol. 46, no. 5, (2016).

[14] J. N. Deng, "Research on IP and optical fusion network control technology based on software defined architecture", Master's degree thesis of Beijing University of Posts and Telecommunications, vol. 3, (2015).

[15] S. J. Ma and D. Y. Hu, "Implementation of dynamic traffic control for multi service based on IP fusion switching technology based on SDN", Science in China: Information Science, vol. 5, (2016).

[16] J. B. Rong and W. F. Li, "Prospects for the key technologies of the fifth generation communication", Guangdong communication technology, vol. 7, (2015).

[17] S. Nirjon, A. Nicoara, C. H. Hsu, J. Singh and J. Stankovic, "MultiNets: policy oriented real-time switching of wireless interfaces on mobile devices", In: IEEE RTAS'12, (2012).

[18] X. Jin, "SoftCell: scalable and flexible cellular core network architecture", In: Proceedings of the ninth ACM conference on Emerging networking experiments and technologies, (2013).

[19] H. Ali-Ahmad, "CROWD: an SDN approach for DenseNets. Proceedings of the 2013 Second European Workshop on Software Defined Networks Publisher", IEEE Computer Society Bibliometrics, (2013).

[20] K. Nagaraj and S. Katti, "ProCel: smart traffic handling for a scalable software EPC", In: ACM HotSDN'14 HotSDN '14: Proceedings of the third workshop on Hot topics in software defined networking, (2014).

[21] M. Mendonca, K. Obraczka and T. Turletti, "The Case for software defined networking in heterogeneous networked environments", In: ACM CoNEXT Student'12, (2012).

[22] H. Ali-Ahmad, C. Cicconetti, A. Oliva, V. Mancuso, M. R. Sama, P. Seite and S. Shanmugalingam, "An SDN-based network architecture for extremely dense wireless networks. 32013 Software Defined Networks for Future Networks and Services (SDN4FNS), (2013). 
[23] A. Rasha, A. Hassan, M. David and G. Estevez, "LTE-WiFi Carrier Aggregation for Future 5G Systems: A Feasibility Study and Research Challenges", the 9th International Conference on Future Netwoks and Communications(FNC-2014), procedia Computer Science, (2014).

[24] T. Alvin, C. David, H. K. Kae, A. Ivan and K. D. Wong, "Dynamic Backhaul Sensitive Network Selection Scheme in LTE-WiFi Wireless HetNet", Mobile and Wireless Networks, 2013 IEEE 24th International Symposium on Personal, Indoor and Mobile Radio Communications, (2013).

[25] Y. Dong and M. X. Li, "Research of Imaging Interpretation Model of CAT Logging Data", International Journal of Heat and Technology, vol. 34, no. 1, (2016), pp. 47-50.

[26] G. Cannistraro, M. Cannistraro, A. Cannistraro, A. Galvagno and G. Trovato, "Reducing the Demand of Energy Cooling in the CED, "Centers of Processing Data", with Use of Free-Cooling Systems", International Journal of Heat and Technology, vol. 34, no. 3, (2016), pp. 498-502.

[27] S. Arlimatti, S. Hassan and A. Habbal, "Minimization of Communication Cost between Controller Domains with Graph Partitioning Algorithms", Revista de la Facultad de Ingeniería, vol. 31, no. 5, (2016), pp. 01-08

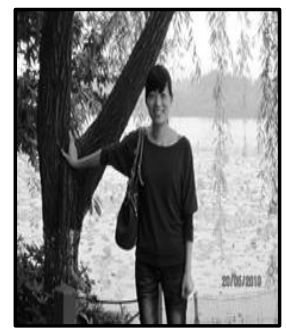

\section{Authors}

Chen Yange, she graduated from Henan Polytechnic University, and is a lecturer in College of Information Engineering, Xuchang University, Henan Province, where she is teaching wireless communication network and network courses. Her area of research is the next generation Internet, Software-Defined Networking (SDN), Internet of things.

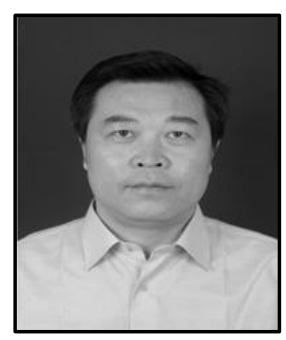

Zhang Zhili, he graduated from South China University of Technology, and is a Professor in College of Information Engineering, Xuchang University, Henan Province, where he is teaching network courses. His area of research is the next generation Internet, Software-Defined Networking (SDN). 